\title{
The relationship between right-to-left shunt and brain white matter lesions in Japanese patients with migraine: a single center study
}

\author{
Akio Iwasaki ${ }^{1 *}$, Keisuke Suzuki ${ }^{2}$, Hidehiro Takekawa ${ }^{1,3}$, Ryotaro Takashima ${ }^{2}$, Ayano Suzuki ${ }^{1}$, Shiho Suzuki ${ }^{2}$
} and Koichi Hirata ${ }^{2}$

\begin{abstract}
Background: There may be a link between right-to-left shunt (RLs) and brain white matter lesions (WMLs) in patients with migraine. In this study, we assessed the relationship between WMLS and RLs in Japanese migraine patients.

Methods: A total of 107 consecutive patients with migraine with (MA) and without aura (MWOA) were included in this study. Contrast transcranial Doppler ultrasound was used to detect RLs. WMLs were graded using brain magnetic resonance imaging based on well-established criteria.

Findings: The prevalence of RLs was significantly increased in the WMLs positive group ( $n=24)$ compared with the WMLs negative group ( $n=83$ ) (75.0\% vs. 47.0\%, $p=0.015)$. In prevalence of WMLs between MA and MWOA patients, there were no statistical differences $(p=0.410)$. Logistic regression analysis adjusted by age and disease duration of migraine identified an RLs-positive status as the sole determinant for the presence of WMLs $(O R=6.15 ; 95 \% \mathrm{Cl}$ 1.82-20.8; $p=0.003)$
\end{abstract}

Conclusion: Our study suggests a possible link between RLs and WMLs in Japanese patients with migraine.

Keywords: Migraine, Right-to-left shunt, Brain white matter lesions, Patent foramen ovale, Pulmonary arteriovenous malformations, Transcranial ultrasonography

\section{Background}

Patients with migraine have been reported to exhibit a two- to fourfold increase in WMLs compared to subjects without migraine [1, 2]. Igarashi et al. [3] reported a significantly increased prevalence of WMLs in patients with migraine compared to controls.

Also, a significant relationship between PFO and migraine has been reported [4]. In a recent meta-analysis, PFO is associated with 2.5 -fold increased prevalence for migraine and 3.4-fold increased prevalence migraine with aura [5]. In a cross sectional study, we have found increased prevalence of right-to-left shunts (RLs) $(62.9 \%$ vs. $44.0 \%)$ and PFO (54.8\% vs. $30.0 \%)$ in patients with

\footnotetext{
* Correspondence: a.iwasaki.n@gmail.com

${ }^{1}$ Stroke Division, Department of Neurology, Dokkyo Medical University, 880

Kitakobayashi, Mibu, Shimotsuga-gun, Tochigi 322-0293, Japan

Full list of author information is available at the end of the article
}

migraine with aura (MA) compared with those with migraine without aura (MWOA) in Asian populations [6]. Although the exact mechanisms of association between migraine and PFO remain unclear, activation of the trigeminal nerve and cerebrovascular system by the passage of metabolic substances or subclinical emboli through a PFO has been implicated [7]. In addition, several reports have described a positive relationship between RLs and brain white matter lesions (WMLs) [8]. Both PFO and pulmonary arteriovenous malformation (pAVM) are known risk factors for cryptogenic ischemic stroke in young subjects. Furthermore, elderly individuals with severe WMLs are at risk for developing stroke [9], and migraine has also been associated with an increased risk of stroke [10].

These observations suggest a possible link between migraine, RLs and WMLs. However, the association 
between RLs and WMLs has not been well studied in Asian populations.

We conducted a single-center, cross-sectional study consisting of consecutive migraine patients from a headache outpatient clinic to test our hypothesis that the presence of WMLs in migraine patients is associated with RLs.

\section{Methods}

Between April 2014 and May 2016, migraine patients were recruited from our headache outpatient clinic at the Department of Neurology of Dokkyo Medical University Hospital. In Japan, unlike in Europe and America, there is no formal system of medical referral. A referral is recommended but not necessary: in fact, most of the patients who visited to our headache outpatient clinic were not referral cases [11]. MA and migraine without aura (MWOA) were diagnosed by headache specialists (RT, SS and $\mathrm{KH}$ ) according to the International Classification of Headache Disorders, 3rd edition (beta version) [12]. Clinical information, including smoking; onset age of migraine; family history of migraine; sensitivity to light, sound or smell; and comorbid diseases such as hypertension, dyslipidemia, diabetes mellitus, and atrial fibrillation was obtained by questioning the patients. No patient had atrial fibrillation. Clinical characteristics and WMLs were compared between RLs-positive and RLs-negative groups.

RLs was assessed in all the patients by transcranial Doppler ultrasound (TCD) (Pioneer TC8080 System, Nicolet Vascular, TCD system, Tokyo, Japan) with intravenous injection of agitated saline with microbubbles by trained neurologists (AI, HT and AS). The details of measurements and settings were described elsewhere [6].

At first, prior to intravenous injection of contrast agent, simple observation was performed for $20 \mathrm{~min}$. Then, contrast agent was injected intravenously during the Valsalva maneuver. If high intensity transient signals (HITS) were detected after $10 \mathrm{~s}$ Valsalva load release, PFO was diagnosed [13-15]. If HITS were detected without Valsalva load, PFO or pAVM was diagnosed. Large shunts were defined as greater than 26 HITS, and middle shunts were defined as 5-26 HITS. The same procedure was repeated 3 times for all the patients.

Patients underwent 1.5-tesla brain magnetic resonance imaging (MRI) (Symphony, Sonata, Siemens Japan Company, Tokyo, Japan). Fluid-attenuated inversion recovery (FLAIR) images $(\mathrm{TR}=10,000 \mathrm{msec}, \mathrm{TE}=98 \mathrm{msec}, \mathrm{IT}=$ $2,500 \mathrm{msec}$ ) with the axial or frontal section images of $5 \mathrm{~mm}$ slices were obtained to evaluate the extent of WMLs in the deep white matter. WMLs were defined as lesions in the deep or subcortical white matters with faint high signals on T2-weighted images, clearly high intensity on FLAIR images and iso-intensity or slightly low signals on T1-weighted signals that were distinguishable from perivascular spaces. Two trained neurologists (HT and KS) who were blinded to clinical information, including the presence of RLs, graded brain WMLs according to a previously published grading score [16]. Briefly, WMLs were classified into the following five grades: Grade 0, absent; Grade 1, $<3 \mathrm{~mm}$ lesions, boundary sharp; Grade 2, >3 mm lesions; Grade 3, confluent foci on deep white matter; Grade 4, confluence widely distributed throughout most of the white matter. The presence of WMLs was defined as Grade 2 or greater in this study. Representative MRI images of patients are shown in Fig. 1.

\section{Statistical analysis}

All data are described as proportions (\%) and medians (range) or means $( \pm \mathrm{SD})$. The patients were classified into the WML-positive or WML-negative group. Univariate analyses were conducted to compare characteristics between the two groups. The chi-square test and Mann-Whitney $U$ test were used to compare characteristics between the WML-positive group and WML-negative groups. Based on the results, logistic regression was conducted to evaluated contributing factors to WMLs positive. All $p$ values were two-tailed, and values $<0.05$ were considered significant. All statistical analysis was performed using IBM SPSS ${ }^{\circledast}$ for Mac, version 23 (Tokyo, Japan).

The institutional review board of Dokkyo Medical University Hospital approved the study. All patients provided written informed consent to participate.

\section{Findings}

Total 119 consecutive migraine patients $(39.8 \pm 13.0$ years, 8 men and 111 women) were enrolled. Twelve patients were excluded from the study: 6 because of a loss in permeability during TCD evaluation of temporal bones, 2 due to insufficient information about migraine and 4 in whom MRI scans were not feasible because of claustrophobia or disagreement. Finally, 107 patients (95.5\%) were included in this study (Fig. 2). Ten patients (9.3\%) were diagnosed with chronic migraine (headache frequency of 15 or more days per month for more than 3 months). Of the total patients, 49 patients $(45.8 \%)$ used preventive medicine for migraine.

\section{Detection of RLs}

A total of 107 subjects (women 101, median age 39.0 years, range 14-74 years) underwent TCD examination (Table 1). When contrast agent injection with the Valsalva maneuver was performed, HITs were detected in 57 subjects (53.3\%). With contrast agent administration at rest, HITs were detected in 11 subjects (10.7\%). Based on these results, a total of 46 subjects (43.0\%) were diagnosed with PFO and 11 subjects (10.7\%) were diagnosed with PFO or pAVM. The maximum numbers of HITs per single examination were as follows: 1-5 

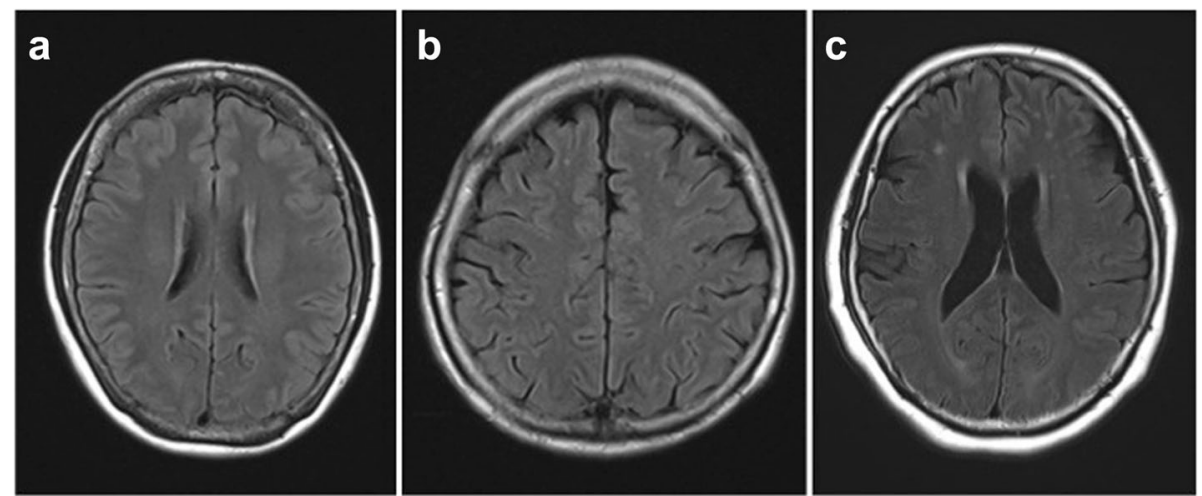

Fig. 1 Grading of brain white matter lesions. a, grade 0 (absent); b grade 1 ( $<3 \mathrm{~mm}$ lesions, boundary sharp); and $\mathbf{c}$, grade 2 ( $>3 \mathrm{~mm}$ lesions)

HITs, 37 subjects; 5-26 HITs, 7 subjects; greater than 26 HITs, 13 subjects.

\section{Comparison of factors between WML-positive and WML- negative patients}

Of 107 subjects, 24 patients $(22.4 \%)$ had brain WMLs, all of which were grade 2 . No one exhibited grade 3 or 4 WMLs. There was no statistical difference in the severity of WMLs between patients with MWA and MWOA. None of chronic migraine patients had WMLs. The rate of preventive medicine use did not differ between the WML-positive and WML-negative groups $(p=0.997)$.

The prevalence of RLs and PFO in the WML-positive group was significantly higher than that in the WMLnegative group (RLs, $75.0 \%$ vs $47.0 \%, p=0.015$; PFO, $62.5 \%$ vs $37.3 \%, p=0.028$ ) (Fig. 3). Regarding the ratio of patients with MA or MWOA, there was no differences prevalence between the WML-positive group and WML-negative groups $(p=0.410)$. The WML-positive group was significantly older than the WML-negative group $(p<0.001)$. Disease duration for migraine was also longer in the WML-positive group (median: 29.5 years) than the WML-negative group (median: 17.0 years) ( $p=$ 0.001). With the exception of RLs, age and disease duration, there were no differences in characteristics between the WML-positive and WML-negative groups (Table 2).

Based on the results of univariate analysis, multivariate analysis was conducted to adjust for age and disease duration of migraine that may have influenced WML prevalence. Multivariate logistic regression analysis indicated that the presence of RLs were independently associated with the presence of WMLs $(p=0.003$; OR $=$ 6.15; 95\% CI 1.82-20.8).

\section{Discussion}

In our study, we investigated the prevalence of RLs and the relationship between clinical background factors, type of migraine and WMLs.

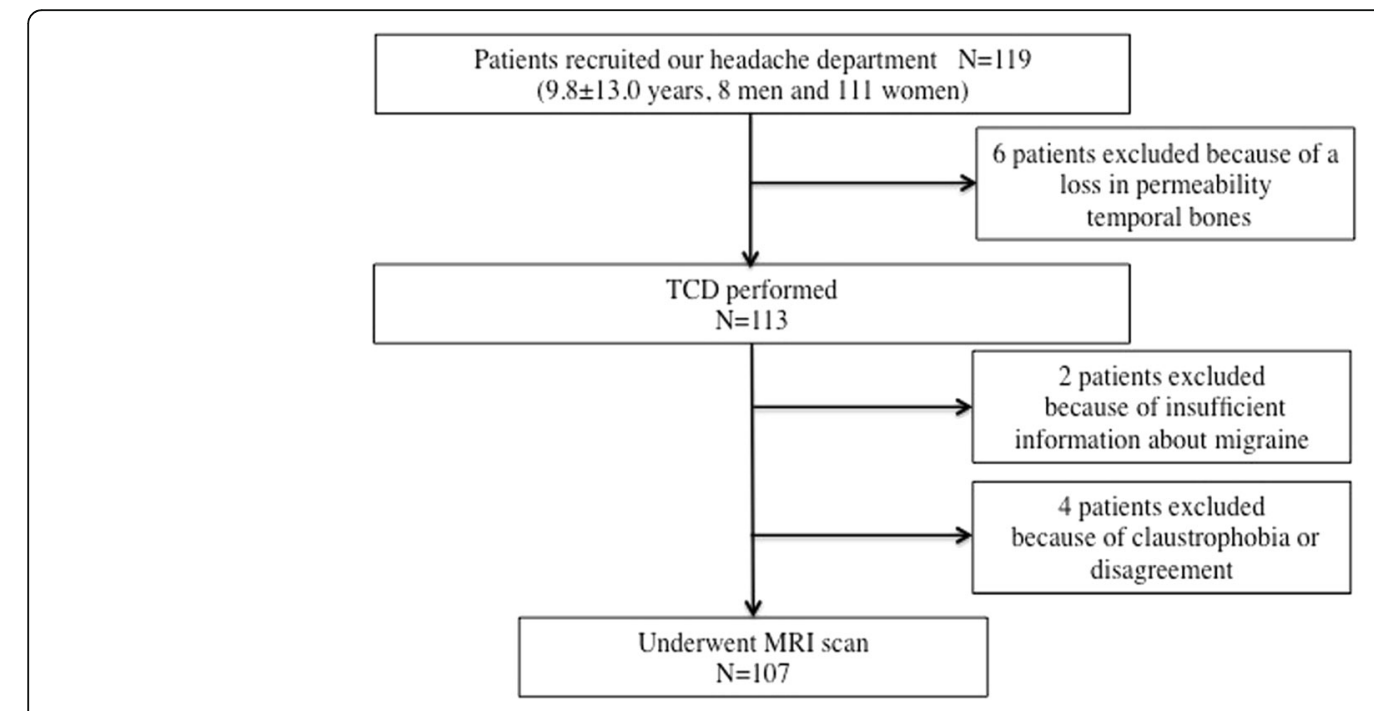

Fig. 2 Flow chart of patient enrolment 
Table 1 Prevalence of RLS and patient characteristics

\begin{tabular}{ll}
\hline Characteristics & Total $(n=107)$ \\
\hline RLs positive, $\mathrm{n}(\%)$ & $57(53.3)$ \\
PFO, $\mathrm{n}(\%)$ & $46(43.0)$ \\
PFO or pulmonary arteriovenous & $11(10.3)$ \\
malformations suspected, $\mathrm{n}(\%)$ & $13(12.1)$ \\
Large shunt, $\mathrm{n}(\%)$ & $39.0(14-74)$ \\
Age, median (range) years & $6(5.6)$ \\
Sex, male, $\mathrm{n}(\%)$ & $59(55.1)$ \\
Aura, $\mathrm{n}(\%)$ & $8(7.5)$ \\
Hypertension, $\mathrm{n}(\%)$ & $3(2.8)$ \\
Diabetes mellitus, $\mathrm{n}(\%)$ & $11(10.3)$ \\
Dyslipidemia, $\mathrm{n}(\%)$ & $7(6.5)$ \\
Smoking, $\mathrm{n}(\%)$ & $71(66.4)$ \\
Family history of migraine, $\mathrm{n}(\%)$ & $81(75.7)$ \\
Photophobia, $\mathrm{n}(\%)$ & $79(73.8)$ \\
Phonophobia, $\mathrm{n}(\%)$ & $60(56.1)$ \\
Hypersensitivity to smell, $\mathrm{n}(\%)$ & $11(10.3)$ \\
Altered taste, $\mathrm{n}(\%)$ & $7(6.5)$ \\
Allodynia, $\mathrm{n}(\%)$ & $18.0(9-40)$ \\
Onset age of migraine, median & \\
(range) years & \\
\hline
\end{tabular}

MA Migraine with aura, MWOA Migraine without aura, RLs Right to left shunts Chi-square test and Mann-Whitney $U$ test

An increased prevalence in WMLs in the RLs-positive group compared to the RLs-negative group was observed. Furthermore, the presence of RLs was an independent predictor for the presence of WMLs in multivariate regression analysis. Aging and vascular risk factors, such as hypertension, diabetes mellitus, and dyslipidemia, have been associated with WMLs in individuals without migraine [17-19]; however, these findings were not supported by our study. Our findings suggest a significant relationship between RLs and WMLs in

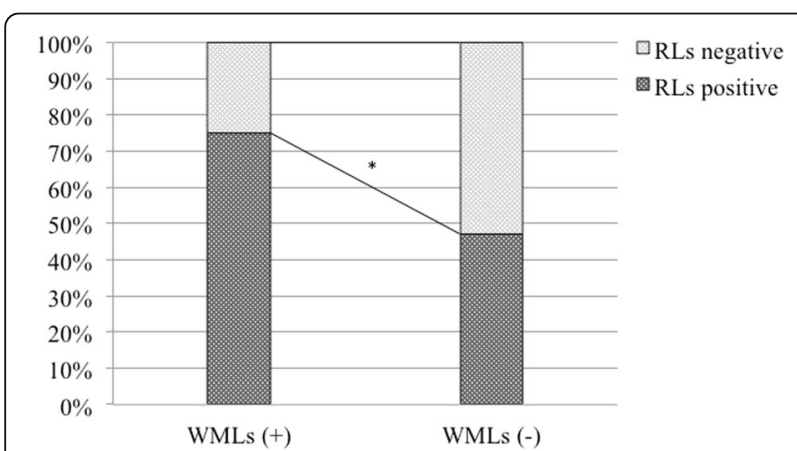

Fig. 3 Prevalence of RLs between WML-positive group and WMLsnegative group. ${ }^{*} p=0.015$. RLs: Right to left shunts, WMLs: White matter lesions
Japanese patients with migraine. Several studies have reported an increased prevalence of WMLs in patients with migraine compared to individuals without headaches after controlling for vascular factors $[1-3,20]$. In a study by Igarashi et al. [3], an increased prevalence of WMLs was found in Japanese patients with migraine, but WMLs were not associated with headache severity or disease duration. In our study, none of chronic migraine patients had WMLs, suggesting headache frequency does not seem to be an important factor for WMLs.

Several studies showed migraine, especially migraine with aura to be a risk factor for WMLs [21, 22]. Additionally, the risk for ischemic stroke was reported to be higher in patients with migraine with aura than with migraine without aura [23]. In contrast, other studies did not show differences in prevalence of WMLs between MWA and MWOA patients [24]. Zhang et al. [25] described that, although no differences were observed in WMLs in MA and MWOA groups, substantially lower cerebral blood flow found in association with high WMLs load suggests that WMLs in MWA may be associated with alteration in resting cerebral blood flow. In our study, we did not observe any differences in WMLs between MA and MWOA groups. However, it is possible that other factors, such as L-arginine, dimethylarginine levels [24] and D-dimer levels as well as headache attack frequencies [26], might have affected WMLs independent of the presence or absence of auras.

Several studies analyzing cohorts of Caucasian patients with migraine did not show a significant association between RLs and WMLs [27, 28]. In contrast, Park et al. [29] showed that the presence of RLs is an independent predictor for the prevalence of small, but not medium or large, WMLs based on multivariate analysis of 425 subjects (242 patients with migraine and 183 patients with tension-type headache). The authors speculated that small paradoxical emboli might have caused small WMLs, triggering migraine headaches. In agreement with the referenced study, our current study showed a significant increase in WMLs prevalence in the RLspositive group compared with the RLs-negative group, supporting a possible link between RLs and WMLs in migraine. Our results and those from Park et al.'s study [29] suggest that RLs may be associated with small, but not large, WMLs in Asian patients with migraine.

Although the pathogenesis associated with WMLs in patients with migraine is unclear, subclinical ischemia, blood brain barrier permeability, reactive astrocytic gliosis-induced injury and endothelial dysfunction may have a role, as well as RLs [4, 20]. Regarding the mechanism underlying the potential relationship between PFO and migraine, PFO may allow vasoactive chemicals, such as serotonin and endothelin, or embolic material to bypass the pulmonary filter and reach the cerebral 
Table 2 Comparison of characteristics between WMLs positive and negative group

\begin{tabular}{|c|c|c|c|}
\hline Characteristics & WMLs $(+)(n=24)$ & WMLs $(-)(n=83)$ & $p$ value \\
\hline RLs positive, $\mathrm{n}(\%)$ & $18(75.0)$ & $39(47.0)$ & 0.015 \\
\hline PFO, n (\%) & $15(62.5)$ & $31(37.3)$ & 0.028 \\
\hline Large shunt, n (\%) & $5(20.8)$ & $8(9.6)$ & 0.139 \\
\hline Age, median (range), years & $46.5(24-74)$ & $36.0(14-65)$ & $<0.001$ \\
\hline Sex, male, n (\%) & $0(0)$ & $6(7.2)$ & 0.175 \\
\hline Migraine with aura (MA), n (\%) & $15(62.5)$ & $44(53.0)$ & 0.410 \\
\hline Hypertension, n (\%) & $2(8.3)$ & $6(7.3)$ & 0.856 \\
\hline Diabetes mellitus, n (\%) & $1(4.2)$ & $2(2.4)$ & 0.646 \\
\hline Dyslipidemia, n (\%) & $2(8.3)$ & $9(10.8)$ & 0.721 \\
\hline Smoking, n (\%) & $1(4.2)$ & $6(7.2)$ & 0.593 \\
\hline Family history of migraine, $\mathrm{n}(\%)$ & $16(66.7)$ & $55(66.3)$ & 0.971 \\
\hline Photophobia, n (\%) & $19(79.2)$ & $62(74.7)$ & 0.653 \\
\hline Phonophobia, n (\%) & $18(75.0)$ & $61(73.5)$ & 0.882 \\
\hline Hypersensitivity to smell, n (\%) & $15(62.5)$ & $45(54.2)$ & 0.471 \\
\hline Altered taste, n (\%) & $5(20.8)$ & $6(7.2)$ & 0.053 \\
\hline Allodynia, n (\%) & $2(8.3)$ & $5(6.0)$ & 0.687 \\
\hline Duration of migraine, median (range) years & $29.5(0-52)$ & $17.0(0-51)$ & 0.001 \\
\hline Onset age of migraine, median (range), years & $17.5(9-37)$ & $18.0(4-40)$ & 0.625 \\
\hline
\end{tabular}

MA Migraine with aura, MWOA Migraine without aura, RLs right-to-left shunt, WMLs white matter lesions, NS not significant

Chi-square test and Mann-Whitney $U$ test

$<0.05$ is significantly italicize

circulation to induce a migraine attack [30, 31]. Additionally, paradoxical air microemboli through the PFO may induce cerebral electrical activity, triggering migraine attacks [32]. In addition, these vasoactive chemicals may play important role in increased WMLs. Several studies found that migraine is associated to several vascular disorders, such as stroke or ischemic heart diseases [33]. In some cases, repeated small paradoxical embolisms may result in an increase in WMLs.

The current study has several limitations. First, not all patients with migraine who were seen in our hospital underwent TCD examination and MRI scanning; therefore, selection bias might have affected the results. The male to female ratio was lower in our study (1:16) than that in a population-based study of migraine $191 \mathrm{pa}-$ tients in Japan (1:3.6) [34], which may have caused by selection bias. Because of small sample size of male patients, we could not evaluate difference in characteristics including WMLs between male and female migraine patients.

Second, embolic risk factors, such as D-dimer levels, use of oral contraceptives and past history of pregnancy and recurrent miscarriage were not evaluated. Oral contraceptives are well known as a risk factor of stroke in migraine and non-migraine subjects [35]. According to a study of 146 parous and nulliparous women [36], history of pregnancy was not related to the presence of WMLs; however, PFO was not evaluated. Thus, further evaluation of these factors is needed. These factors might have affected the prevalence of WMLs due to silent paradoxical cerebral infarctions.

\section{Conclusions}

RLs were found in over half of a cohort of Japanese patients with migraine. Our study suggests a possible link between RLs and WMLs in Japanese migraine patients.

\section{Abbreviations}

FLAIR: Fluid-attenuated inversion recovery; HITS: High-intensity transient signals; MA: Migraine with aura; MCA: Middle cerebral artery; MRI: Magnetic resonance imaging; MWOA: Migraine without aura; PAVM: pulmonary arteriovenous malformation; PFO: Patent foramen ovale; RLs: Right to left shunts; TCD: transcranial Doppler; WMLs: White matter lesions

\section{Acknowledgments}

We thank all of the medical staff for their assistance with this study.

\section{Funding}

None.

\section{Authors' contributions}

Al contributed to the study concept and design, the diagnosis of the patients and the acquisition, analysis and interpretation of data and drafted the manuscript. KS and HT contributed to the study concept and design, the diagnosis of the patients, the acquisition, analysis and interpretation of data and the manuscript revision. RT, AS, SS contributed to the study concept and design and data acquisition. $\mathrm{KH}$ contributed to the study concept and 
design and supervised the study. All authors read and approved the final manuscript.

\section{Competing interests}

The authors declare that they have no competing interests.

\section{Author details}

${ }^{1}$ Stroke Division, Department of Neurology, Dokkyo Medical University, 880 Kitakobayashi, Mibu, Shimotsuga-gun, Tochigi 322-0293, Japan. Department of Neurology, Dokkyo Medical University, Tochigi, Japan. ${ }^{3}$ Center of Medical Ultrasonics, Dokkyo Medical University, Tochigi, Japan.

\section{Received: 6 November 2016 Accepted: 12 December 2016}

Published online: 07 January 2017

\section{References}

1. Kruit MC, van Buchem MA, Hofman PA, Bakkers JT, Terwindt GM, Ferrari MD et al (2004) Migraine as a risk factor for subclinical brain lesions. JAMA 291(4):427-34. doi:10.1001/jama.291.4.427

2. Swartz RH, Kern RZ (2004) Migraine is associated with magnetic resonance imaging white matter abnormalities: a meta-analysis. Arch Neurol 61(9): 1366-8. doi:10.1001/archneur.61.9.1366

3. Igarashi H, Sakai F, Kan S, Okada J, Tazaki Y (1991) Magnetic resonance imaging of the brain in patients with migraine. Cephalalgia 11(2):69-74

4. Schwedt TJ, Demaerschalk BM, Dodick DW (2008) Patent foramen ovale and migraine: a quantitative systematic review. Cephalalgia 28(5):531-40. doi:10.1111/j.1468-2982.2008.01554.x

5. Takagi H, Umemoto T, Group A (2016) A meta-analysis of case-control studies of the association of migraine and patent foramen ovale. J Cardiol 67(6):493-503. doi:10.1016/j.jjcc.2015.09.016

6. Iwasaki A, Suzuki K, Takekawa H, Takashima R, Suzuki A, Suzuki S et al (2016) Prevalence of right to left shunts in Japanese patients with migraine: a single-center study. Intern Med. in press.

7. Sathasivam S, Sathasivam S (2013) Patent foramen ovale and migraine: what is the relationship between the two? J Cardiol 61(4):256-9. doi:10.1016/j.jjcc. 2012.12.005

8. Liu JR, Plotz BM, Rohr A, Stingele R, Jansen O, Alfke K (2009) Association of right-to-left shunt with frontal white matter lesions in T2-weighted MR imaging of stroke patients. Neuroradiology 51(5):299-304. doi:10.1007/ s00234-009-0496-9

9. Etminan M, Takkouche B, Isorna FC, Samii A (2005) Risk of ischaemic stroke in people with migraine: systematic review and meta-analysis of observational studies. BMJ 330(7482):63. doi:10.1136/bmj.38302.504063.8F

10. Vermeer SE, Hollander M, van Dijk EJ, Hofman A, Koudstaal PJ, Breteler MM et al (2003) Silent brain infarcts and white matter lesions increase stroke risk in the general population: the Rotterdam Scan Study. Stroke 34(5):1126-9. doi:10.1161/01.STR.0000068408.82115.D2

11. Suzuki S, Suzuki K, Miyamoto M, Miyamoto T, Watanabe $Y$, Takashima R et al (2011) Evaluation of contributing factors to restless legs syndrome in migraine patients. J Neurol 258(11):2026-35. doi:10.1007/s00415-011-6064-3

12. Headache Classification Committee of the International Headache S (2013) The International Classification of Headache Disorders, 3rd edition (beta version). Cephalalgia 33(9):629-808. doi:10.1177/0333102413485658

13. Blersch WK, Draganski BM, Holmer SR, Koch HJ, Schlachetzki F, Bogdahn U et al (2002) Transcranial duplex sonography in the detection of paten foramen ovale. Radiology 225(3):693-9. doi:10.1148/radiol.2253011572

14. Jauss M, Zanette E (2000) Detection of right-to-left shunt with ultrasound contrast agent and transcranial Doppler sonography. Cerebrovasc Dis 10(6): 490-6, doi:16119

15. Ringelstein EB, Droste DW, Babikian VL, Evans DH, Grosset DG, Kaps M et al (1998) Consensus on microembolus detection by TCD. International Consensus Group on Microembolus Detection. Stroke 29(3):725-9

16. Shinohara Y, Tohgi H, Hirai S, Terashi A, Fukuuchi Y, Yamaguchi T et al (2007) Effect of the Ca antagonist nilvadipine on stroke occurrence or recurrence and extension of asymptomatic cerebral infarction in hypertensive patients with or without history of stroke (PICA Study). 1. Design and results at enrollment. Cerebrovasc Dis 24(2-3):202-9. doi:10.1159/000104478

17. Cohen Jl, Cazettes F, Convit A (2011) Abnormal Cholesterol is Associated with Prefrontal White Matter Abnormalities among Obese Adults: a Diffusion Tensor Imaging Study. Neuroradiol J 24(6):854-61
18. Vuorinen M, Solomon A, Rovio S, Nieminen L, Kareholt I, Tuomilehto J et al (2011) Changes in vascular risk factors from midlife to late life and white matter lesions: a 20-year follow-up study. Dement Geriatr Cogn Disord 31(2):119-25. doi:10.1159/000323810

19. Xiong YY, Mok V (2011) Age-related white matter changes. J Aging Res 2011:617927. doi:10.4061/2011/617927

20. Tana C, Tafuri E, Tana M, Martelletti P, Negro A, Affaitati G et al (2013) New insights into the cardiovascular risk of migraine and the role of white matter hyperintensities: is gold all that glitters? J Headache Pain 14:9. doi:10.1186/1129-2377-14-9

21. Kruit MC, van Buchem MA, Launer LJ, Terwindt GM, Ferrari MD (2010) Migraine is associated with an increased risk of deep white matter lesions, subclinical posterior circulation infarcts and brain iron accumulation: the population-based MRI CAMERA study. Cephalalgia 30(2):129-36. doi:10.1111/j.1468-2982.2009.01904.x

22. Dinia L, Bonzano L, Albano B, Finocchi C, Del Sette $M$, Saitta L et al (2013) White matter lesions progression in migraine with aura: a clinical and MRI longitudinal study. J Neuroimaging 23(1):47-52. doi:10.1111/j.1552-6569.2011.00643.x

23. Spector JT, Kahn SR, Jones MR, Jayakumar M, Dalal D, Nazarian S (2010) Migraine headache and ischemic stroke risk: an updated meta-analysis. Am J Med 123(7):612-24. doi:10.1016/j.amjmed.2009.12.021

24. Erdelyi-Botor S, Komaromy H, Kamson DO, Kovacs N, Perlaki G, Orsi G et al (2016) Serum L-arginine and dimethylarginine levels in migraine patients with brain white matter lesions. Cephalalgia. doi:10.1177/0333102416651454

25. Zhang Q, Datta R, Detre JA, Cucchiara B (2016) White matter lesion burden in migraine with aura may be associated with reduced cerebral blood flow. Cephalalgia. doi:10.1177/0333102416649760

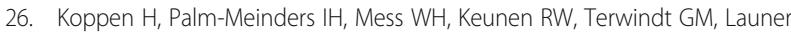
$L J$ et al (2016) Systemic right-to-left shunts, ischemic brain lesions, and persistent migraine activity. Neurology 86(18):1668-75. doi:10.1212/WNL. 0000000000002538

27. Adami A, Rossato G, Cerini R, Thijs VN, Pozzi-Mucelli R, Anzola GP et al (2008) Right-to-left shunt does not increase white matter lesion load in migraine with aura patients. Neurology 71(2):101-7. doi:10.1212/01.wnl. 0000316798.25510.f2

28. Calviere L, Tall P, Massabuau P, Bonneville F, Larrue V (2013) Migraine with aura and silent brain infarcts lack of mediation of patent foramen ovale. Eur J Neurol 20(12):1560-5. doi:10.1111/ene.12240

29. Park HK, Lee SY, Kim SE, Yun CH, Kim SH (2011) Small deep white matter lesions are associated with right-to-left shunts in migraineurs. J Neurol 258(3):427-33 doi:10.1007/s00415-010-5771-5

30. Dalla Volta G, Guindani M, Zavarise P, Griffini S, Pezzini A, Padovani A (2005) Prevalence of patent foramen ovale in a large series of patients with migraine with aura, migraine without aura and cluster headache, and relationship with clinical phenotype. J Headache Pain 6(4):328-30. doi:10.1007/s10194-005-0223-9

31. Borgdorff P, Tangelder GJ (2012) Migraine: possible role of shear-induced platelet aggregation with serotonin release. Headache 52(8):1298-318. doi:10.1111/j.1526-4610.2012.02162.x

32. Sevgi EB, Erdener SE, Demirci M, Topcuoglu MA, Dalkara T (2012) Paradoxical air microembolism induces cerebral bioelectrical abnormalities and occasionally headache in patent foramen ovale patients with migraine. J Am Heart Assoc 1(6):e001735. doi:10.1161/JAHA.112.001735

33. Sacco S, Ornello R, Ripa P, Tiseo C, Degan D, Pistoia F et al (2015) Migraine and risk of ischaemic heart disease: a systematic review and meta-analysis of observational studies. Eur J Neurol 22(6):1001-11. doi:10.1111/ene.1270

34. Sakai F, Igarashi H (1997) Prevalence of migraine in Japan: a nationwide survey. Cephalalgia 17(1):15-22

35. Schurks M, Rist PM, Bigal ME, Buring JE, Lipton RB, Kurth T (2009) Migraine and cardiovascular disease: systematic review and meta-analysis. BMJ 339: b3914. doi:10.1136/bmj.b3914

36. Postma IR, de Groot JC, Aukes AM, Aarnoudse JG, Zeeman GG (2014) Cerebral white matter lesions and perceived cognitive dysfunction: the role of pregnancy. Am J Obstet Gynecol 211(3):257 e1-5. doi:10.1016/j.ajog.2014.02.031 